\title{
LuQi Formula Regulates NLRP3 Inflammasome to Relieve Myocardial-Infarction-Induced Cardiac Remodeling in Mice
}

\author{
Xiaoqing Zhang ${ }^{1},{ }^{1}$ Dandan Zhao, ${ }^{1}$ Jiling Feng, ${ }^{1}$ Xiaoli Yang, ${ }^{1}$ Zhenzhen Lan, ${ }^{1}$ Tao Yang, \\ Xiaoni Kong, ${ }^{2}$ Huiyan Qu $\mathbb{D}^{1}{ }^{1}$ and Hua Zhou ${ }^{1}{ }^{1}$ \\ ${ }^{1}$ Department of Cardiovascular Disease, ShuGuang Hospital Affiliated to Shanghai University of Traditional Chinese Medicine, \\ Shanghai, China \\ ${ }^{2}$ Central Laboratory, ShuGuang Hospital Affiliated to Shanghai University of Traditional Chinese Medicine, Shanghai, China
}

Correspondence should be addressed to Huiyan Qu; sgyyjxb@163.com and Hua Zhou; zhouhua@shutcm.edu.cn

Received 26 February 2021; Revised 4 June 2021; Accepted 17 June 2021; Published 28 June 2021

Academic Editor: Daniel Dias Rufino Arcanjo

Copyright (c) 2021 Xiaoqing Zhang et al. This is an open access article distributed under the Creative Commons Attribution License, which permits unrestricted use, distribution, and reproduction in any medium, provided the original work is properly cited.

Background. Excessive activation of the nod-like receptor family pyrin domain containing 3(NLRP3) inflammasome plays a significant role in the progression of cardiac injury. In China, it has been well recognized that Chinese herbal medicine is markedly effective in treating cardiovascular diseases (CVDs). LuQi Formula (LQF) has been used clinically for more than 10 years and confirmed to be effective in improving cardiac function and inhibiting apoptosis. However, the specific mechanisms underlying its efficacy are mostly unknown. This study aimed to evaluate whether LQF could alleviate cardiac injury and apoptosis by regulating the NLRP3 inflammasome and the caspase-3/Bax pathway. Purpose. In this study, we investigated the effects of LQF on cardiac remodeling in a mouse model of myocardial infarction (MI) in vivo. Methods. Forty male C57BL/6 mice were randomly divided into four groups: the sham group, the model group, the LQF group, and the perindopril group, with a sample size $(n)$ of 10 mice in each group. Except the sham group, the other groups received left anterior descending (LAD) coronary artery ligation to induce MI and then treated with LQF, perindopril, or saline. Six weeks after MI, echocardiography was used to evaluate cardiac structure and function. Myocardial tissue morphology was observed by haematoxylin and eosin (H\&E) staining, and heart samples were stained with Masson's trichrome to analyse myocardial fibrosis. Myocardial hypertrophy was observed by fluorescent wheat germ agglutinin (WGA) staining. The expressions of NLRP3, ASC, Cle-caspase-1, IL-1 $\beta$, TXNIP, Cle-caspase-3, Bcl-2, and Bax in heart tissues were assessed by western blot analysis. mRNA expressions of ANP and BNP in heart tissues were measured by RT-PCR. The expression of reactive oxygen species in myocardial tissue was detected by using a DCFH-DA probe. Results. Echocardiographic analysis showed that compared with the model group, the left ventricular ejection fraction (LVEF) and left ventricular fractional shortening (LVFS) in the LQF and perindopril group were increased $(P<0.05)$, left ventricular internal diameter end diastole (LVIDd) and left ventricular internal diameter end-systole (LVIDs) were reduced $(P<0.05)$, and H\&E and Masson's trichrome staining of cardiac tissues showed that LQF and perindopril could partially reverse ventricular remodeling and alleviate myocardial fibrosis $(P<0.05)$. WGA fluorescence results showed that compared with the model group, myocardial hypertrophy was significantly reduced in the LQF and perindopril group. We also found that LQF and perindopril reduce the oxidative stress response in the heart of MI mice. The protein expression of NLRP3, ASC, Cle-caspase-1, IL- $1 \beta$, TXNIP, Cle-caspase-3, and Bax was downregulated in the LHF and perindopril treatment group, and Bcl-2 expression was upregulated. Conclusion. LQF and perindopril significantly attenuated cardiac injury and apoptosis in the MI model. In addition, we found that LQF effectively inhibited the activation of the NLRP3/ASC/caspase-1/IL-1 $\beta$ cascade, decreased inflammatory infiltration, delayed ventricular remodeling, and downregulated caspase-3/Bax signaling, which can effectively reduce the apoptosis of cardiomyocytes. Perindopril showed the same mechanism. 


\section{Introduction}

Chronic heart failure ( $\mathrm{CHF}$ ), characterized by high mortality and morbidity, is the final outcome of many CVDs [1]. Myocardial infarction (MI) is one of the most important causes of heart failure (HF), and how to reduce cardiac dysfunction and HF risk after MI has always been the focus of CVD research [2].

Growing evidence suggests that an aseptic inflammatory response during acute myocardial ischemia (AMI) plays a crucial role in the formation of scar and remodeling of the damaged myocardium [3]. An early inflammatory response might increase matrix degradation, causing the infarcted myocardial tissue to heal and form a scar. However, the aggravation, prolongation, or extension of the inflammatory response might lead to the deterioration of the remodeling and dysfunction after MI [4]. Therefore, moderate regulation of the inflammatory response after $\mathrm{MI}$ is very important for myocardial remodeling of the left ventricle to prevent $\mathrm{HF}$ [5]. Targeted inhibition of most of the induced inflammatory responses could be a potential treatment for MI.

Many studies have found that activation of the NLRP3 protein leads to an enhanced inflammatory response and cardiac dysfunction and remodeling, which aggravates acute myocardial injury in MI animals $[6,7]$. In contrast, a lack of NLRP3 inflammasome components might reduce the inflammatory response and promote cardioprotective effect [8]. The NLRP3 inflammasome is a supramolecular protein complex that activates caspase- 1 and modulates the maturation of interleukin $1 \beta$ (IL-1 $\beta$ ), thereby initiating the inflammatory response [9]. Recent research has shown that NLRP3 plays an important role in CVDs [10]. Gao et al. found that targeting the NLRP3 inflammasome can effectively reduce myocardial fibrosis and cardiac remodeling after MI [11]. Wang et al.'s study has shown that NLRP3 inflammasome is strongly associated with CVB3-induced myocarditis, and in the first 7 days after CVB3 infection, the level of IL- $1 \beta$, cleaved caspase-1, and ASC was increased [12]. Therefore, targeted inhibition of NLRP3 activation could reduce the inflammatory response after MI and delay ventricular remodeling.

Traditional Chinese medicine (TCM) has been widely used in the prevention and treatment of diseases over the years and has been clinically validated as a good means to promote health. In China, it has been well recognized that TCM is markedly effective to treat CHF. LQF, an effective prescription to treat $\mathrm{CHF}$, has been used widely in clinical practice; LQF (Chinese national patent number ZL2014102933164) is an empirical compound prescription of several common medicinal herbs in TCM, composed of antler, safflower, Astragalus membranaceus, Codonopsis pilosula, Cassia twig, and, Semen lepidii, aiming at "warming kidney and Eliminating Dampness." LQF can effectively relieve chest tightness, edema, and other uncomfortable symptoms of CHF patients. However, its effect on ventricular remodeling remains to be studied. As the cornerstone of clinical treatment of $\mathrm{HF}$, angiotensin-converting enzyme inhibitors (ACEIs) improve ventricular remodeling $[13,14]$. However, whether ACEIs improve ventricular remodeling in mice with $\mathrm{HF}$ in association with downregulation of NLRP3 inflammation remains unclear. Therefore, this study aimed to assess the effects of LQF and the long-acting ACEI perindopril on ventricular remodeling in mice with HF.

\section{Materials and Methods}

2.1. Animals and Drugs. Male wild-type (WT) C57BL/6J mice (body weight $20-23 \mathrm{~g}$ ), aged 8 weeks, were obtained from the Experimental Animal Center $(n=10$ for each group), Shanghai University of Chinese Medicine. All the animal experimentation was complied with the ARRIVE guidelines, and this study was conducted in accordance with safe animal testing specifications (Safety Certificate Number: SYXK-HU-2020-0009 and Animal Ethics Code PZSHUTCM200807012). All mice were maintained under a controlled temperature $\left(20 \pm 5^{\circ} \mathrm{C}\right)$ and humidity $(55 \pm 15 \%)$ with a $12 \mathrm{~h}$ light $/ 12 \mathrm{~h}$ dark cycle. LQF dry powder, made by Shanghai Chinese Traditional Pharmaceutical Technology Co., Ltd. (China), was dissolved in distilled water to produce the equivalent of the crude drug concentration of $1.78 \mathrm{~g} / \mathrm{kg}$ and was administered intragastrically. The gavage dose $(1.65 \mathrm{~g} / \mathrm{ml})$ in the present study was determined according to the equivalent patient dose. Perindopril tablets ( $4 \mathrm{mg} / \mathrm{tablet})$ were obtained from Servier Pharmaceutical Co., Ltd. (Tianjin, China) and used as a positive control. Perindopril tablets were crushed and dissolved in double-distilled water at a concentration of $0.06 \mathrm{mg} / \mathrm{ml}$. The sham group mice received water. The starting dose for all mice was from the day after LAD artery ligation, and all mice were treated for 6 weeks.

2.2. Mouse Model of MI. MI surgery was performed as described previously [15]. The mice were anesthetized and mechanically ventilated using a rodent ventilator. Their hearts were exposed by thoracotomy and subsequent pericardiotomy. MI was induced by permanent LAD coronary artery ligation using 7-0 silk suture, and successful induction of AMI was observed when the anterior and apical left ventricular myocardium gradually attained a dull, pale colour with reduced pulse and then confirmed by ST segment elevation on an electrocardiogram. The sham-operated group underwent the same procedure without ligation of the LAD coronary artery.

2.3. Ultrasound Echocardiography. Ultrasound echocardiography was performed by using a Vevo 2100 Imaging System (Visual Sonics Inc., Toronto, ON, Canada) in mice under anesthesia with isoflurane (RWD Life Science Co., Guangdong, China). Briefly, the mice were anesthetized using 1.0-2.5\% isoflurane, and the heart rate was maintained at $\sim 450$ to 550 beats $/ \mathrm{min}$. The heart was examined in the long-axis view at the papillary muscle level, and an M-mode echocardiogram of the mid ventricle was recorded. Cardiac function indices including LVEF, LVFS, LVIDd, and LVIDs were recorded [16]. 
2.4. Western Blotting. Proteins were isolated from the frozen cardiac tissue, and their concentration was calculated using the bicinchoninic acid (BCA) method (Thermo Fisher Scientific, Waltham, MA, USA; A53225). Immunoblotting was performed as previously described [15]. In this study, the primary antibodies used recognized NLRP3 $(1: 1000$, CST, 15101S), Cle-caspase-1 (1:1000, Abcam, ab1789515), ASC $(1: 1000$, CST, 67824), thioredoxin-interacting protein (TXNIP) (1:1000, CST, 14715), caspase-3 (1:1000, CST, 9662), BCL-2 apoptosis regulator (Bcl-2) $(1: 1000$, CST, 3498), IL-1 $\beta$ (1:1000, Abcam, ab234437), $\operatorname{Bax}(1: 1000$, Abcam, ab32503), and glyceraldehyde-3-phosphate dehydrogenase (GAPDH) (1:20000, Proteintech, 60004-1-Ig). Protein bands were visualized using the chemiluminescence system (Tanon Science \& Technology Co., Shanghai, China) for the required time. Quantitative analysis was performed using Image J software (NIH, Bethesda, MD, USA).

2.5. Histological Staining and Evaluation. Heart tissues were isolated, rinsed with phosphate buffered saline (PBS), and fixed in $4 \%$ paraformaldehyde (PFA) over $24 \mathrm{~h}$. Then, the tissues were dehydrated and paraffin-embedded. Next, $5 \mu \mathrm{m}$ thick slices were cut for H\&E staining to explore changes in heart size and for Masson's trichrome staining to visualize fibrosis [17]. After staining, all slices were completely scanned using Caseviewer 2.0 (Pannoramic 250/MIDI, 3DHISTECH, Budapest, Hungary). The severity of cardiac fibrosis was calculated by the Masson's trichrome-positive areas in 4 randomly selected fields per section of cardiac using Image J software.

2.6. Immunohistochemical (IHC) and Immunofluorescence (IF) Analysis. Heart tissues were isolated, rinsed with PBS, and fixed in $4 \%$ PFA over $24 \mathrm{~h}$. The hearts were then dehydrated and paraffin-embedded. Next, $5 \mu \mathrm{m}$-thick slices were cut for IHC and IF analyses. Paraffin sections were dewaxed with water, and antigens were retrieved by heating in sodium citrate buffer. Endogenous peroxidase was removed by adding $30 \% \mathrm{H}_{2} \mathrm{O}_{2}$, and an immunohistochemical pen was used to draw a circle around the tissue. Subsequently, 10\% fetal bovine serum was added to block nonspecific binding to the tissue. The sections were incubated with NLRP 3 and anti-IL- $1 \beta$ primary antibodies overnight at $4^{\circ} \mathrm{C}$. Goat-anti-mouse secondary antibodies were then added and incubated at room temperature for $60 \mathrm{~min}$, followed by washing with PBS three times for $5 \mathrm{~min}$. Then, $30 \mu \mathrm{L}$ of 4', 6diamidino-2-phenylindole (DAPI) solution was added and incubated for $10 \mathrm{~min}$, followed by three PBS washes. For immunofluorescence staining, WGA(GeneTex, USA, GTX01502) was diluted to $0.1 \mathrm{mg} / \mathrm{mL}$, added to the sections, and incubated at room temperature for $2 \mathrm{~h}$. Sections were then washed three times with PBS for $5 \mathrm{~min}$. Then, $30 \mu \mathrm{L}$ of DAPI solution was added to the section and incubated for $10 \mathrm{~min}$, followed by three PBS washes. A fluorescence microscope was used to observe and collect images in a darkroom. Semiquantitative analysis was performed in four randomly selected fields per section using Image J software.
2.7. Terminal Deoxynulceotidyl Transferase Nick-End-Labeling (TUNEL) Assay. Paraffin-embedded cardiac tissues were sectioned into $5 \mu \mathrm{m}$ slices. After dewaxing to water, $20 \mu \mathrm{g} /$ $\mathrm{mL}$ proteinase $\mathrm{K}$ was added to cover the tissue. Twenty minutes later, the sections were washed with PBS three times for $5 \mathrm{~min}$, and TUNEL dye (C1086, Beyotime Biotechnology, Shanghai, China) was added and incubated at $37^{\circ} \mathrm{C}$ for $1 \mathrm{~h}$. The slices were then washed with PBS three times for $5 \mathrm{~min}$. DAPI was added to slices and incubated for $10 \mathrm{~min}$. The slices were sealed with an antifluorescence quenching sealant. Fluorescence microscopy was performed in a darkroom for observation and image collection.

2.8. Reactive Oxygen Species (ROS) Analysis. Heart tissue was separated, washed with PBS, and rapidly frozen in liquid nitrogen. The tissue was then embedded with an optimal cutting temperature (OCT) embedding agent. After embedding, $6 \mu \mathrm{m}$-thick sections were generated using a freezing microtome for ROS staining. Frozen sections were fixed at room temperature with 4\% PFA for 5 minutes and washed three times with PBS for 1 minute each, and then, a tissue pen was used to draw a circle around the tissue. $5 \mu \mathrm{M} 20,70$ dichlorofluorescein diacetate (DCFH-DA) was added to the circled area and incubated at $37^{\circ} \mathrm{C}$ for $20 \mathrm{~min}$, avoiding light, followed by PBS washes. DAPI staining was carried out for $10 \mathrm{~min}$ in the dark at room temperature. Slices were then washed with PBS three times for 5 min each. The slices were sealed with an antifluorescence quenching sealant. Fluorescence microscopy was performed in a darkroom for observation and image collection.

2.9. Malondialdehyde (MDA), Superoxide Dismutase (SOD), and Glutathione (GSH) Analysis. The contents of MDA (A003-1-2, Jiancheng Bioengineering Institute, Nanjing, China), SOD (A001-3-2, Jiancheng Bioengineering Institute), and GSH (A005-1-2, Jiancheng Bioengineering Institute) were determined by colorimetry. The assays were performed according to the manufacturer's recommendations.

2.10. RNA Extraction and RT-PCR. Total RNA was isolated from individual hearts using commercial kits to evaluate mRNA expression of atrial natriuretic peptide (ANP) and brain type natriuretic peptide (BNP). Reverse-transcribed cDNA was obtained using the Total RNA rapid extraction kit (BioTeke, China, RP4002). The primers used to amplify the genes are listed in Table 1. All primer sequences were checked in GenBank to avoid inadvertent sequence homologies. They were designed and synthesized by Sangon Biotechnology (Shanghai, China). Reactions were performed using an SYBR Green PCR master mix (Vazyme, China, Q711-02). Relative amounts of mRNA for specific genes were calculated using the $2{ }^{\Delta \Delta} \mathrm{Ct}$ values [18].

2.11. Statistical Analysis. All results are expressed as the mean \pm standard deviation (SD) and were analyzed using one-way analysis of variance (ANOVA) with the least significant difference- (LSD-) t's multiple comparisons, using 
TABLE 1: PCR primer sequence.

\begin{tabular}{lcc}
\hline Gene name & Primer sequence $\left(5^{\prime}-3^{\prime}\right)$ & $\mathrm{Tm}\left({ }^{\circ} \mathrm{C}\right)$ \\
\hline \multirow{2}{*}{ Anp } & $\mathrm{F5}^{\prime}$ GCTTCCAGGCCATATTGGAG3 & $\mathbf{6 0 . 2}$ \\
& $\mathrm{R}^{\prime}$ GGGGGCATGACCTCATCTT3 & $\mathbf{6 1 . 1}$ \\
\hline \multirow{2}{*}{ Bnp } & F5 $^{\prime}$ GAGGTCACTCCTATCCTCTGG3' & $\mathbf{6 0 . 2}$ \\
& R5 $^{\prime}$ GCCATTTCCTCCGACTTTTCTC $3^{\prime}$ & $\mathbf{6 1 . 2}$ \\
\hline
\end{tabular}

SPSS version 20.0 software (IBM Corp., Armonk, NY, USA). $P<0.05$ was considered statistically significant.

\section{Results}

3.1. LQF Improved Cardiac Function after MI. Cardiac ultrasound is an important method to evaluate cardiac function, and MI results in poor ventricular remodeling [19]. As shown in Figures 1(a)-1(f), in the MI model group, the ejection fraction (EF) and fractional shortening (FS) values were reduced, and LVIDd and LVIDs were augmented compared with the sham group, whereas LQF and perindopril treatment reduced the deterioration of cardiac function and cardiac enlargement. ANP and BNP are sensitive markers of myocardial dysfunction, which correlate positively with the severity of HF. Thus, ANP and BNP are important indicators to determine the progression and prognosis of HF [20]. The qRT-PCR analysis showed that MI-induced increases of Anp and Bnp mRNA expression were partly alleviated in LQF and perindopril mice compared with model mice (Figures $1(\mathrm{~g})$ and $1(\mathrm{~h})$ ). This revealed that LQF and perindopril have protective effects against cardiac function. No significant difference in the cardiac function between the LQF group and the perindopril group was noted.

ACE inhibitor treatment improves morbidity and mortality in post-MI patients [21], and related studies have shown that perindopril can delay myocardial remodeling and myocardial fibrosis. Raj et al. found that resveratrol and perindopril can attenuate cardiac remodeling and contractile dysfunction in rats post infarct [22]. Li et al. have demonstrated that perindopril downregulates Gal-3 and reduces myocardial fibrosis [23]. The intragastric doses of perindopril and LQF in this experiment were both within the safe range, so no obvious adverse reactions occurred during the experiment.

\subsection{LQF Reduced Myocardial Fibrosis and Hypertrophy.} Interstitial fibrosis is the hallmark of cardiac remodeling [24]. To determine whether LQF and perindopril could ameliorate cardiac fibrosis and hypertrophy after MI, we analyzed myocardial injury and fibrosis by $\mathrm{H} \& \mathrm{E}$ and Masson staining. In the sham group, the myocardial fibers were arranged regularly, and there was no exudation, edema, or inflammatory cell infiltration in the myocardial interstitium. No abnormal, blue-stained collagen fibers were found in the myocardial interstitium. In the model group, there were degeneration and edema of cardiomyocytes, uneven staining of myocardial fibers, infiltration of inflammatory cells in the epicardium, staggered distribution of blue-stained collagen fibers, and increased distribution of myocardial fibers. In the LQF group, the myocardial injury was alleviated, the infiltration of inflammatory cells was significantly reduced, and a small amount of blue-stained collagen fibers could be seen. The myocardial morphology of the perindopril group was similar to that of the LQF group (Figures 2(a) and 2(b)).

WGA is an effective indicator of cardiac hypertrophy [25]. As shown in Figures 2(c) and 2(e), compared with the sham group, the cardiomyocytes in the model group were enlarged, the myocardial fiber outline was irregular and deformed, and the number of cells was decreased. Compared with the model group, the volume of cardiomyocytes in LQF and perindopril groups was smaller, the outlines of the myocardial fibers were relatively regular, and the number of cardiomyocytes was increased. Furthermore, the effect of perindopril on reducing cardiomyocyte hypertrophy was more significant.

3.3. LQF Inhibited NLRP3 Inflammasome Activation. An aseptic inflammatory response during MI plays an important role in scar formation and myocardial remodeling. Moderate regulation of the inflammatory response after MI is very important to prevent HF; however, an excessive inflammatory response will lead to the deterioration of myocardial remodeling and dysfunction after MI. Many studies have found that NLRP3 is activated after MI, resulting in an enhanced inflammatory response and cardiac dysfunction [26]. To determine whether the effect of LQF and perindopril on improving cardiac function is related to the inflammasome, we studied the expression of NLRP3 inflammasome in the heart. Unsurprisingly, NLRP3, ASC, Cle-caspase- 1 , and IL- $1 \beta$ protein levels in the cardiac tissue of the mice in the model group were significantly higher than those in the other groups. After treating with LQF and perindopril, mice after MI had reduced levels of NLRP3, ASC, Cle-caspase-1, and IL-1 $\beta$ (Figures 3(a)-3(e)). In addition, immunohistochemical staining further showed that LQF and perindopril could reduce the levels of NLRP3 and IL-1 $\beta$ after MI (Figures 3(f)-3(h)). Moreover, compared with perindopril, LQF showed no significant difference in inhibiting the NLRP3 and IL-1 $\beta$ after MI. These findings indicated that LQF and perindopril can effectively reduce the levels of proteins associated with the NLRP3 inflammasome and cytokine IL- $1 \beta$.

\subsection{LQF Suppressed the Rise of ROS and Reduced the Oxidative} Stress Response in the Hearts of MI Mice. After MI, the necrotic cells and matrix fragments in the infarct area produce damage-associated molecular patterns (DAMPs) signaling molecules, leading to the increase of mitochondrial ROS and activation of NLRP3 inflammasome. To clarify whether the inhibition of the NLRP3 inflammasome by LQF and perindopril is related to the inhibition of ROS, we detected the ROS levels in the cardiac tissue (Figures 4(a) and 4(c)). The ROS staining results showed that a higher level of ROS was observed in the model group. However, LQF and perindopril reduced the production of ROS. By contrast, ROS could powerfully trigger the association of TXNIP with NLRP3. As 

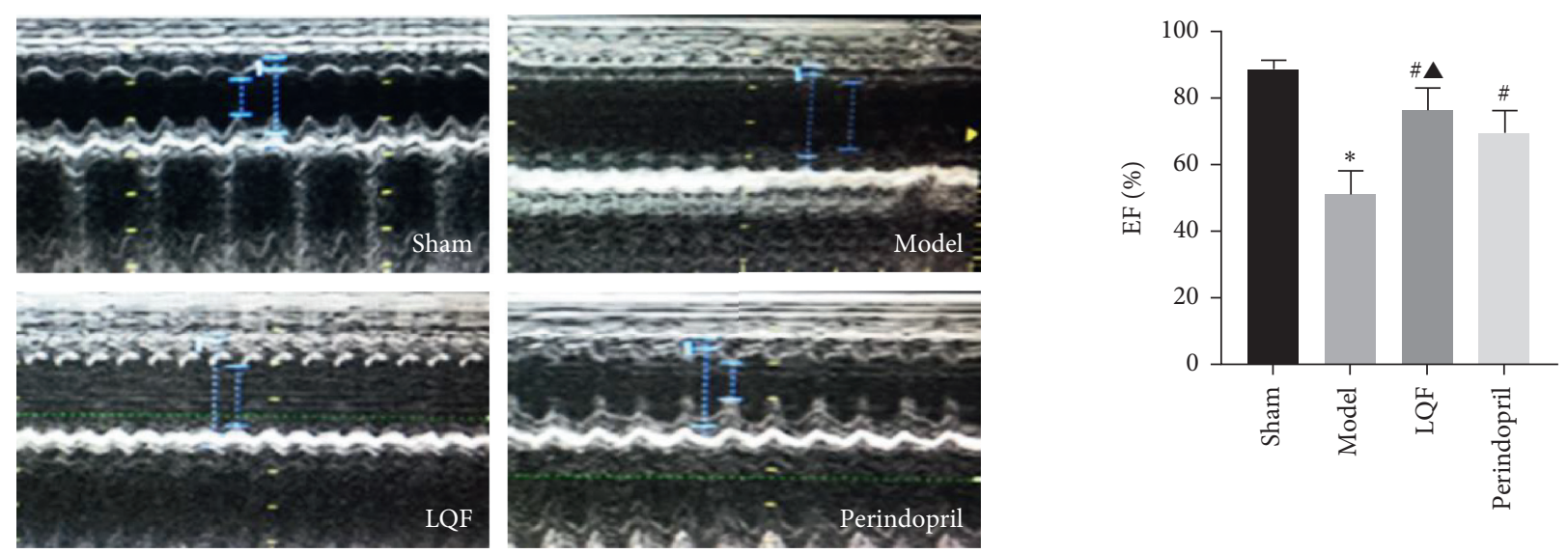

(a)

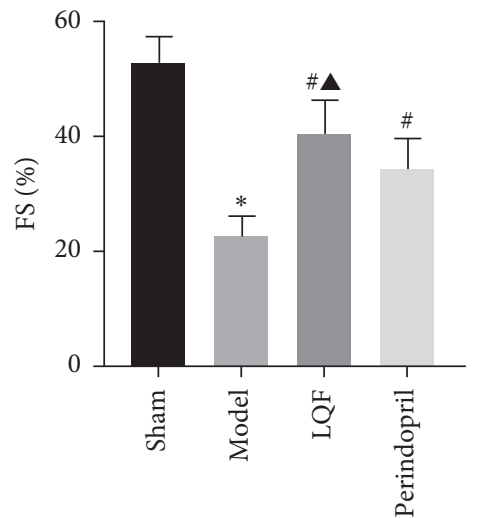

(b)

(c)

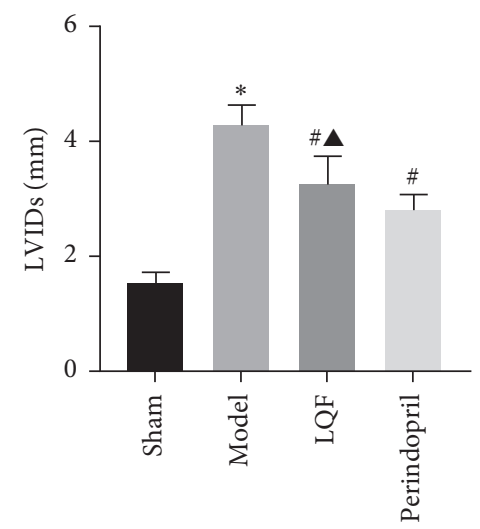

Sham

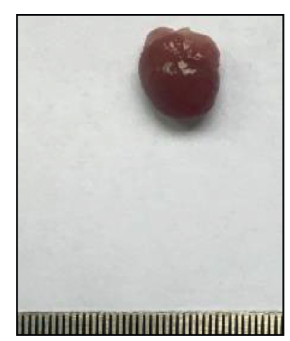

Model

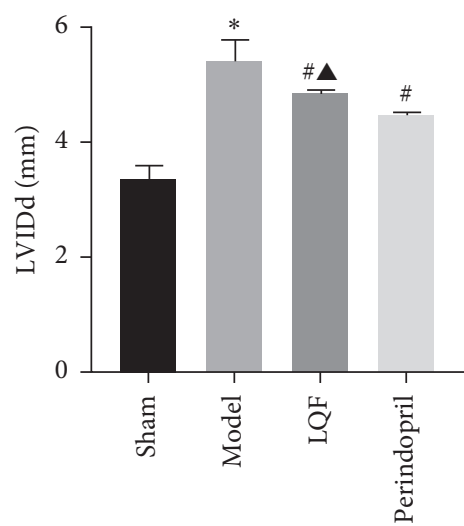

(d)

(e)
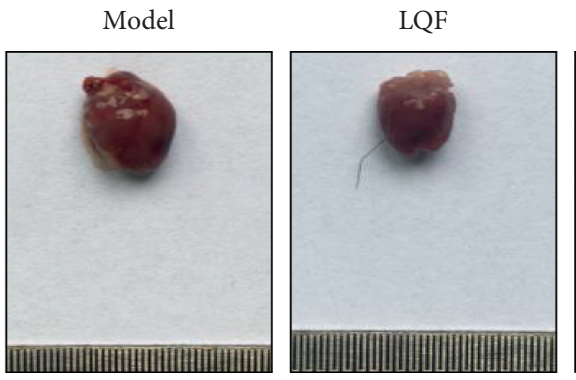

Perindopril

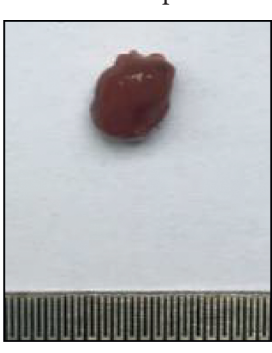

(f)

FIgURE 1: Continued. 


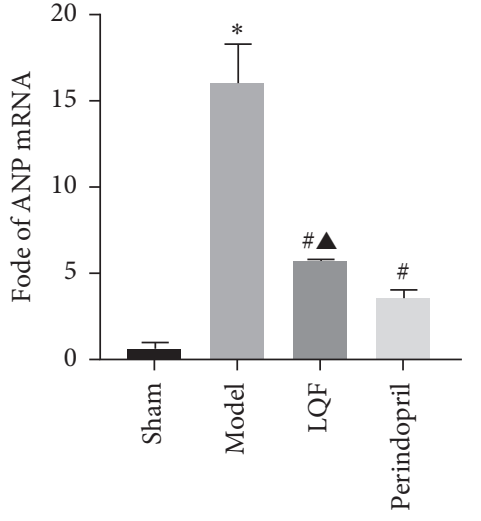

(g)

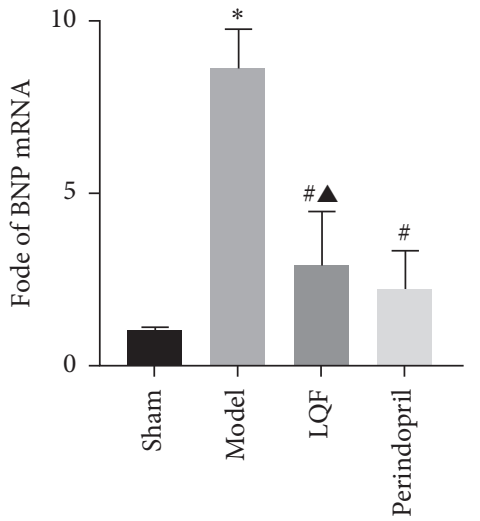

(h)

FIGURE 1: LQF improves cardiac function after MI. (a) Representative images of echocardiography tracings of the mice 6 weeks after MI in four groups. (b) EF\%, (c) FS\%, (d) LVIDd, and (e) LVIDs. (f) Representative gross morphology of whole hearts. (g), (h) Transcript levels of Anp and Bnp, as determined by qRT-PCR. ${ }^{*} P<0.05 v$ s. the sham group, ${ }^{\#} P<0.05 v s$. the model group, and ${ }^{\boldsymbol{\Delta}} P<0.05 v s$. the perindopril group.
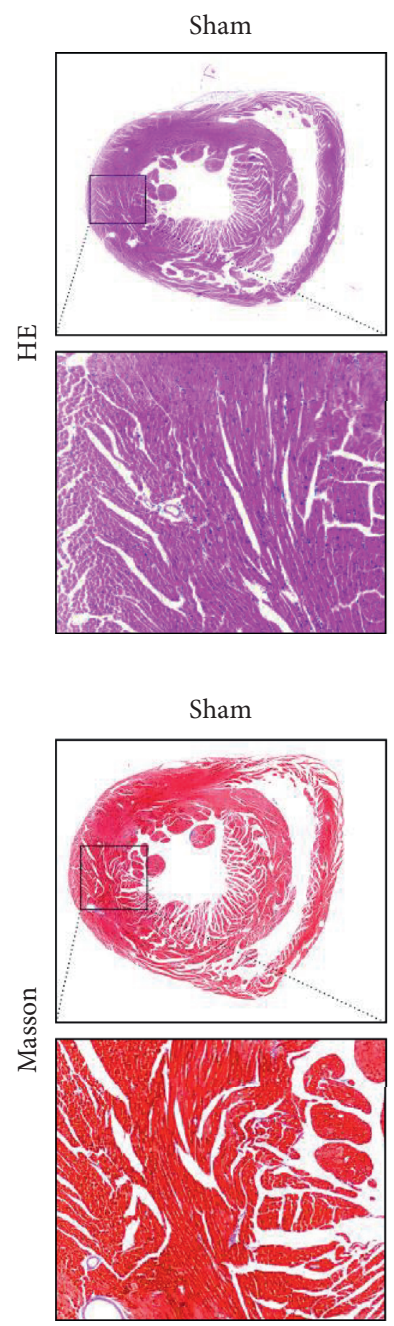

Model
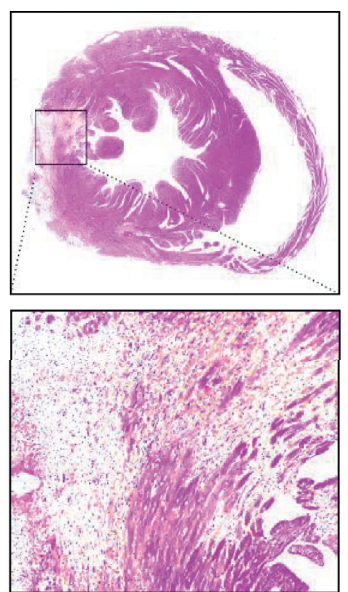

(a)
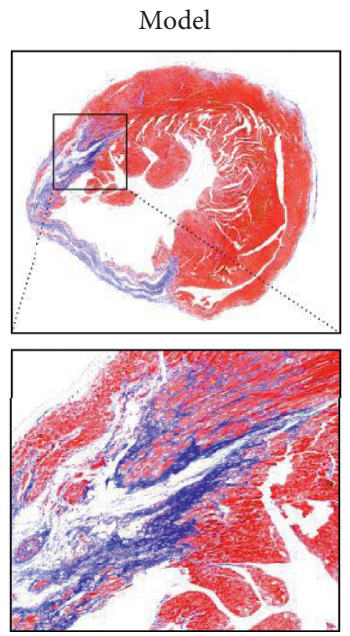

LQF
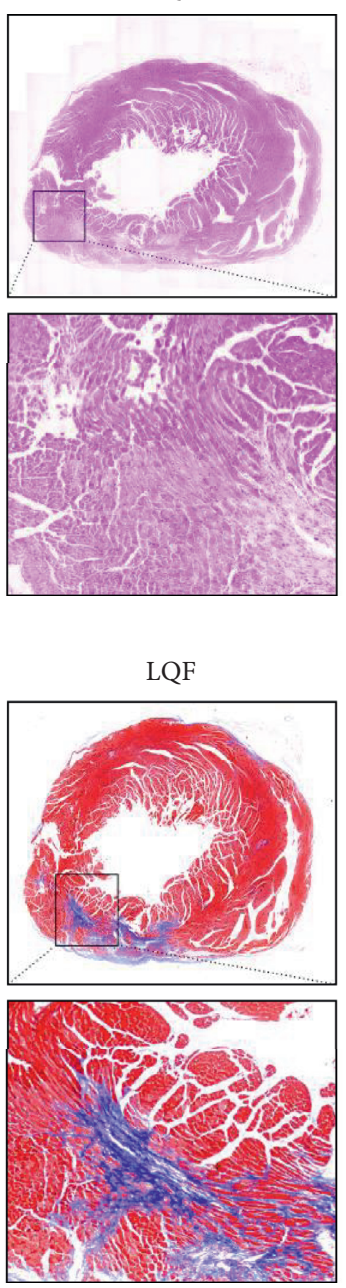
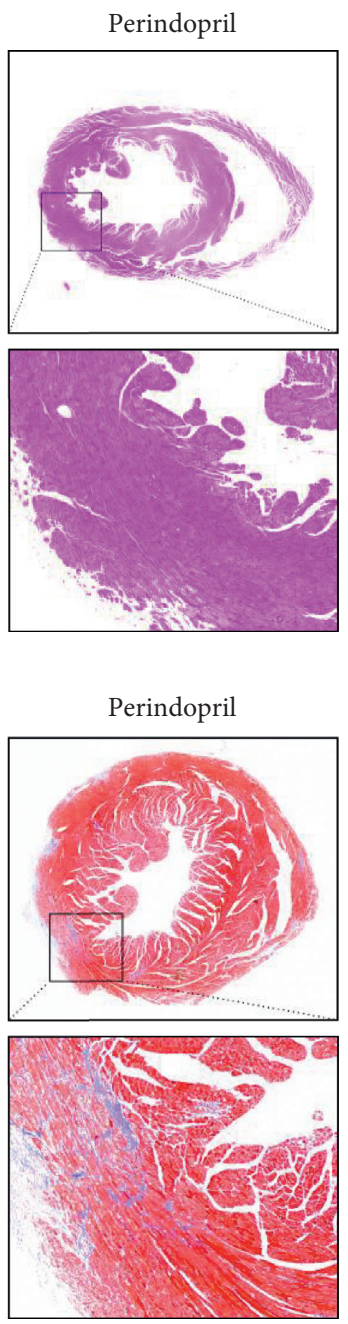

(b)

Figure 2: Continued. 

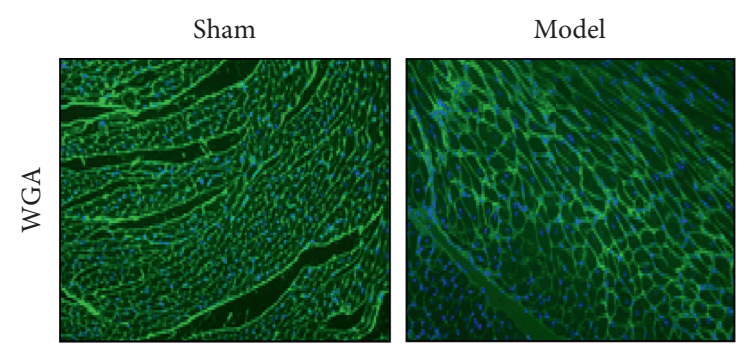

(c)
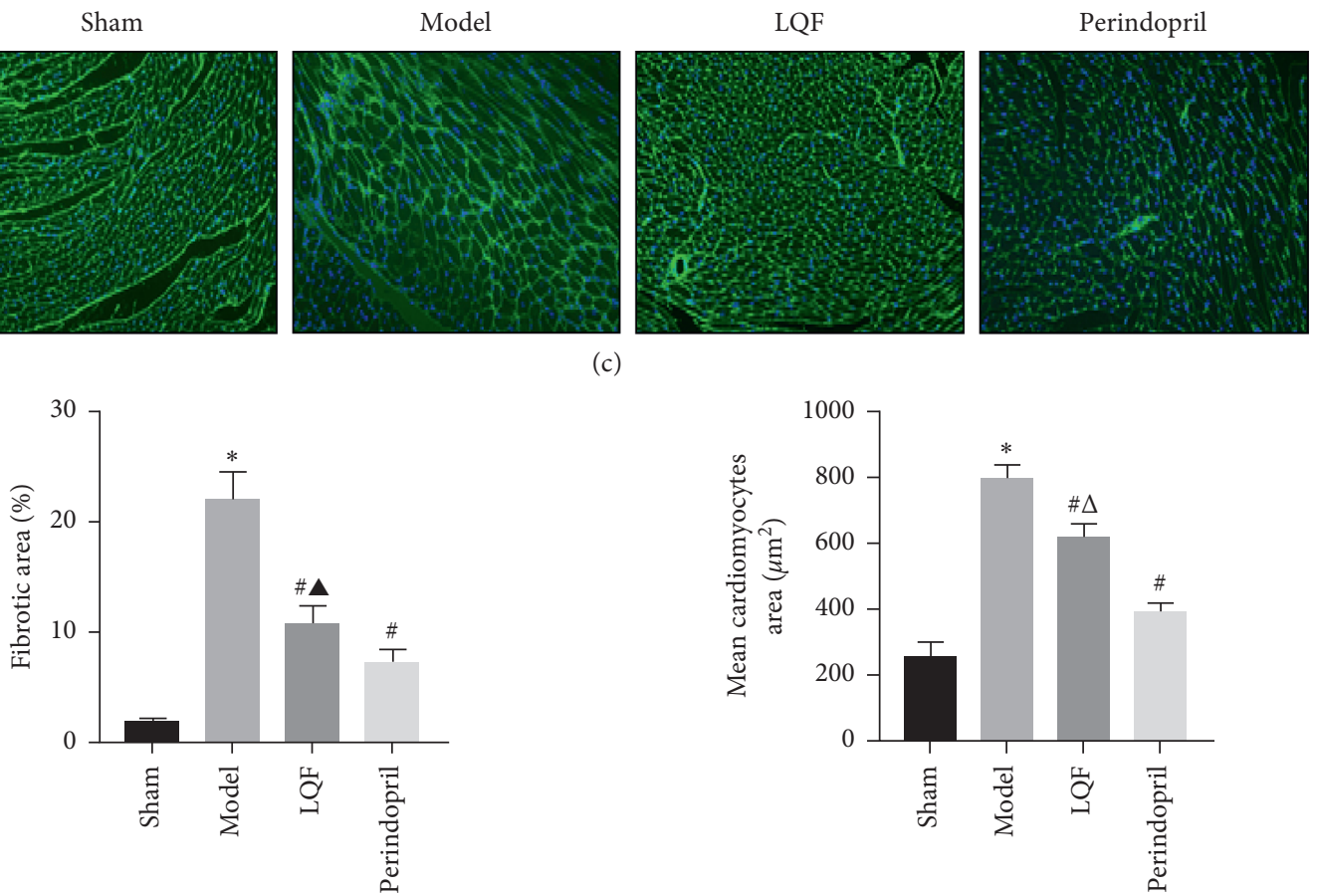

(d)

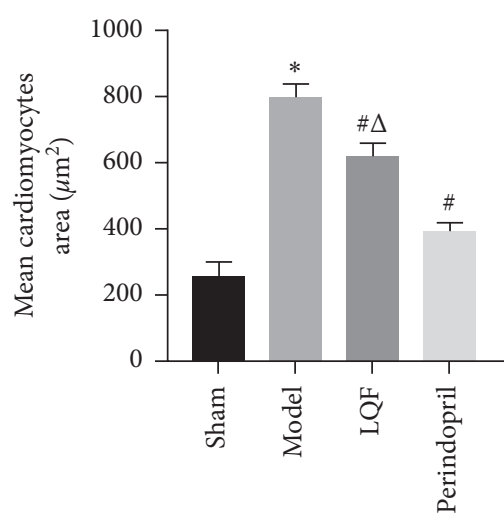

(e)

FIGURE 2: LQF reduces myocardial fibrosis and hypertrophy. (a) Representative image of H\&E staining showing the size of mouse myocytes (the scale bar of the picture above is $1 \mathrm{~mm}$, and the scale bar of the picture below is $100 \mu \mathrm{m})(n=3)$. (b) Representative Masson's trichrome staining of the heart sections (the scale of the picture above is $1 \mathrm{~mm}$, and the scale of the picture below is $100 \mu \mathrm{m})(n=3)$. (c) Representative images of WGA staining of heart sections (magnification $200 \times)(n=3)$. (d) Quantitative analysis of the fibrotic area. (e) Mean cardiomyocytes area. ${ }^{*} P<0.05 v s$. the sham group, ${ }^{\#} P<0.05 v s$. the model group, ${ }^{\boldsymbol{\Delta}} P<0.05 v s$. the perindopril group, and ${ }^{\triangle} P<0.05 v s$. the perindopril group.

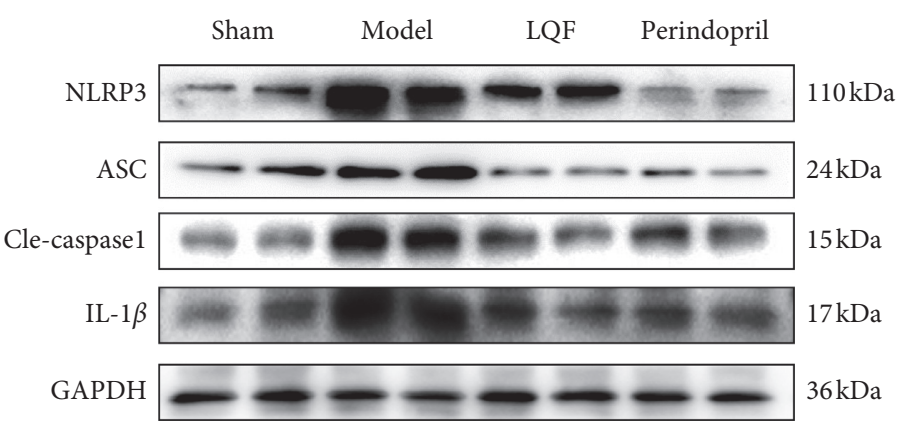

(a)

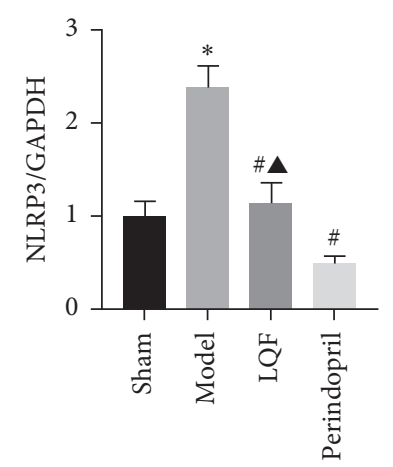

(b)

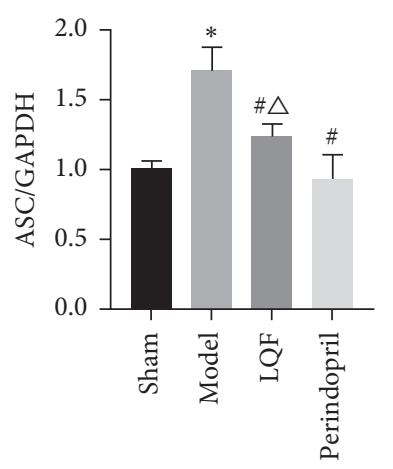

(c)

FIgURE 3: Continued. 


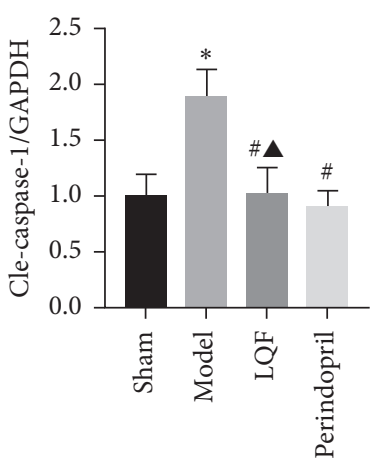

(d)
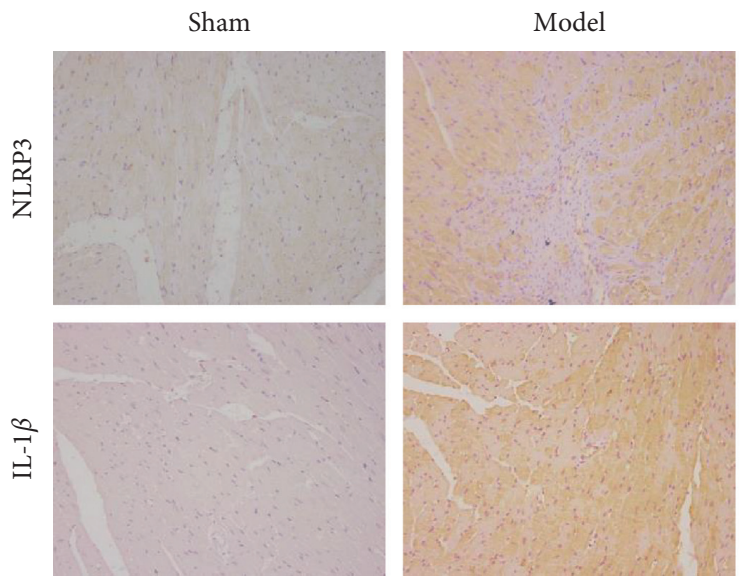

(f)

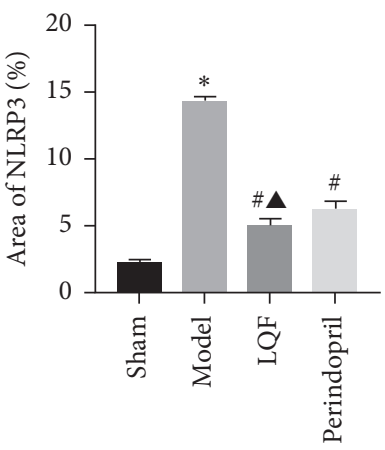

(g)

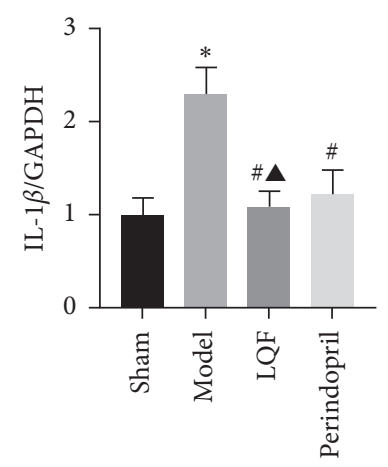

(e)
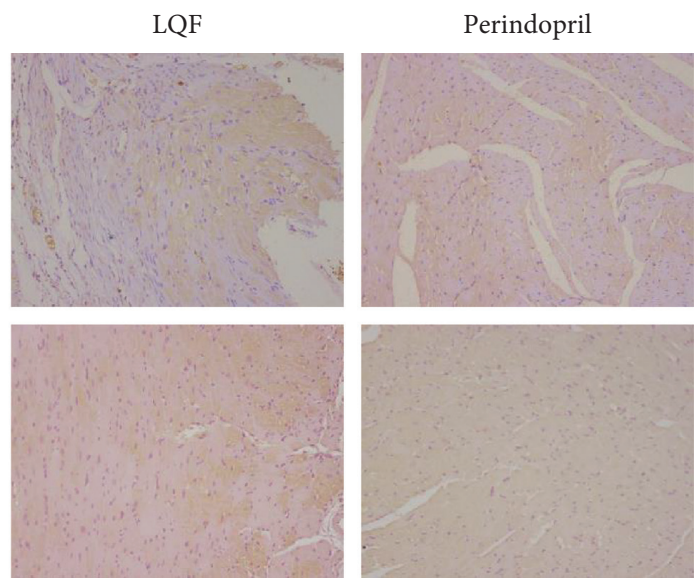

(h)

FIGURE 3: LQF inhibits NLRP3 inflammasome activation. (a)-(e) Representative western blotting and quantification of inflammationrelated proteins, including NLRP3, ASC, Cle-caspase-1, and IL-1 $\beta$, GAPDH served as a loading control $(n=5)$. (f)-(h) Representative image of a cardiac section stained for NLRP3 and IL- $1 \beta$, with the quantification results of IHC (magnification $200 \times)(n=3) .{ }^{*} P<0.05 v s$. the sham group, ${ }^{\#} P<0.05$ vs. the model group, ${ }^{\wedge} P<0.05$ vs. the perindopril group, and ${ }^{\triangle} P<0.05$ vs. the perindopril group.

shown in Figure 4(b), TXNIP levels in the model group were significantly higher than those in the other groups. In the LQF and perindopril groups, TXNIP levels were particularly suppressed (Figure 4(d)). Meanwhile, we assessed levels of SOD, MDA, and GSH (Figures $4(\mathrm{e})-4(\mathrm{~g})$ ), which are all closely related to oxidative stress (OS). The results showed that compared with the sham mice, the level of MDA was significantly increased, while the levels of SOD and GSH were significantly decreased in the model group. Compared with those in the model mice, the level of MDA was significantly decreased, and the levels of SOD and GSH were increased in the LQF group. In addition, SOD and GSH levels in the perindopril group increased more significantly. These results suggest that LQF can repair OS-induced cardiac injury.

3.5. LQF Inhibited Apoptosis in Mice after MI. To identify whether LQF protects the postinfarct myocardium against apoptosis, we assessed apoptotic biomarker levels using western blotting and TUNEL staining. The results show that there was a significant increase in Bcl-2 and a decrease in 

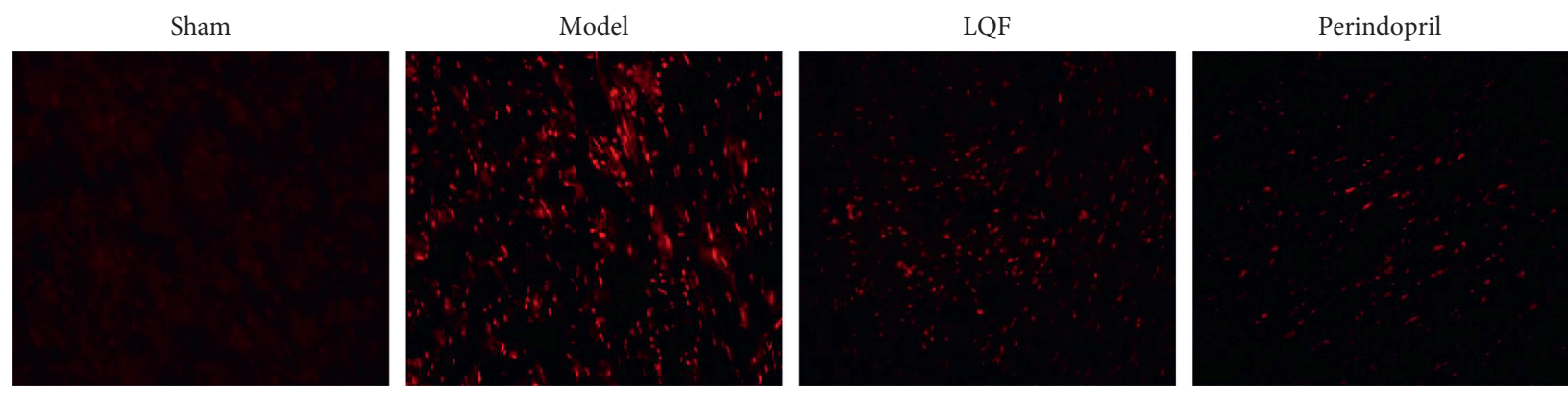

(a)

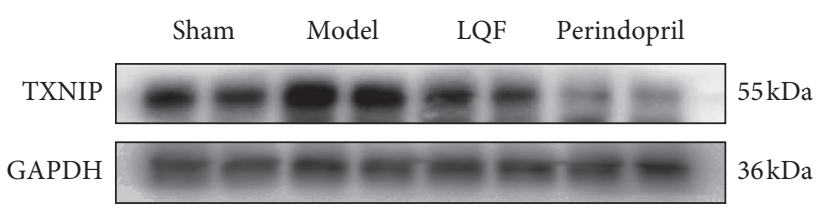

(b)

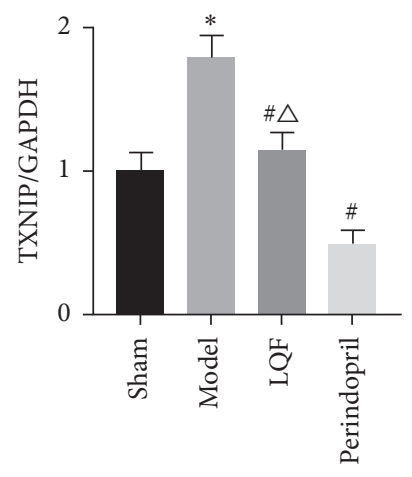

(d)

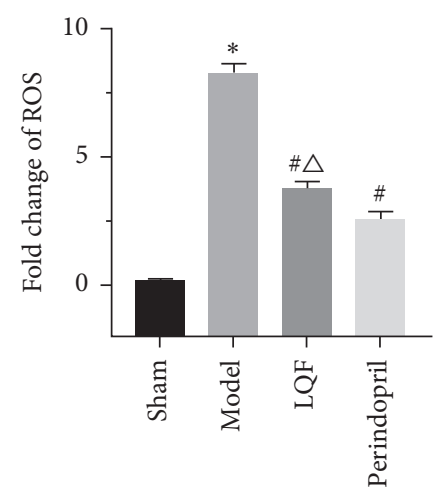

(c)

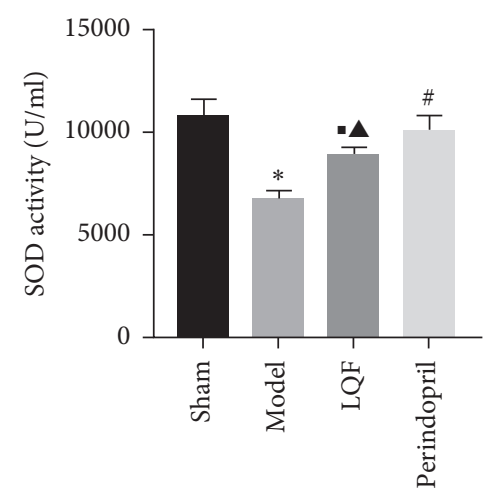

(e)

FIGURE 4: LQF reduces the oxidative stress response in the heart of MI mice. (a) Representative images of ROS in a section of mouse heart assessed using immunofluorescence staining (magnification 200x) $(n=3)$. (b) Representative western blotting of TXNIP ( $n=5)$. (c)-(d) Quantification of the ROS assay and TXNIP. (e)-(g) SOD, MDA, and GSH levels in whole ventricular lysates, as measured using a colorimetric method, ${ }^{*} P<0.05$ vs. the sham group, ${ }^{\#} P<0.05$ vs. the model group, ${ }^{\circledR} P<0.05$ vs. the model group, ${ }^{\triangle} P<0.05$ vs. the perindopril group, and $\boldsymbol{\Delta} P<0.05$ vs. the perindopril group.

Cle-caspase-3 and Bax levels on the border of the MI hearts after LQF and perindopril treatment (Figures 5(a)-5(d)), suggesting that LQF and perindopril have antiapoptotic properties. This result was confirmed by the TUNEL staining. There were dramatically fewer apoptotic (TUNEL-positive) cells on the border of the MI hearts of the LQF and perindopril group compared to that of the model group (Figure 5(e)). According to the results, MI stimulates excess ROS production and activates OS in cardiac tissues, which affects TXNIP activity and prompts TXNIP to bind with NLRP3, further triggering the assembly of the NLRP3 inflammasome. This process continues to activate the NLRP3ASC-caspase-1 axis to release inflammatory factors and trigger cardiac inflammatory responses, which eventually leads to apoptosis of the cardiomyocytes. LQF suppresses the activation of the NLRP3-ASC-caspase-1 axis by inhibiting TXNIP, thus ameliorating cardiac apoptosis.

\section{Discussion}

Chronic heart disease is associated with a persistent lowgrade inflammatory response that promotes adverse cardiac remodeling and is correlated with disease progression [27]. Currently, anti-inflammatory therapies for CVDs, such as those investigated in the Canakinumab Anti-inflammatory Thrombosis Outcomes Study (CANTOS) and Colchicine Cardiovascular Outcomes Trial, have shown effectiveness $[28,29]$. This study demonstrated that MI in mice induced 


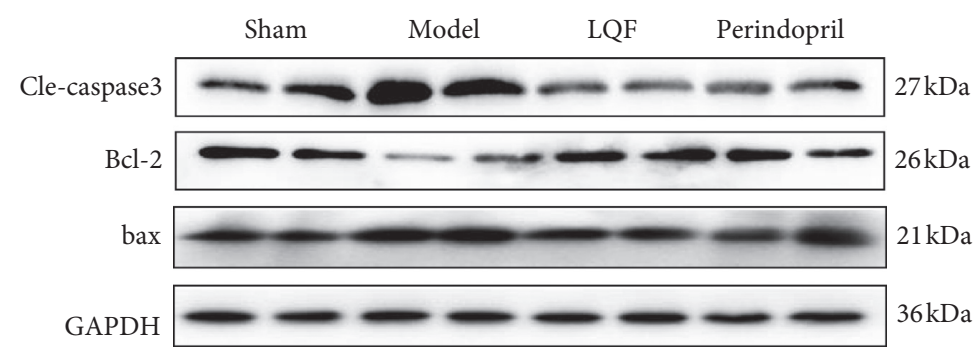

(a)

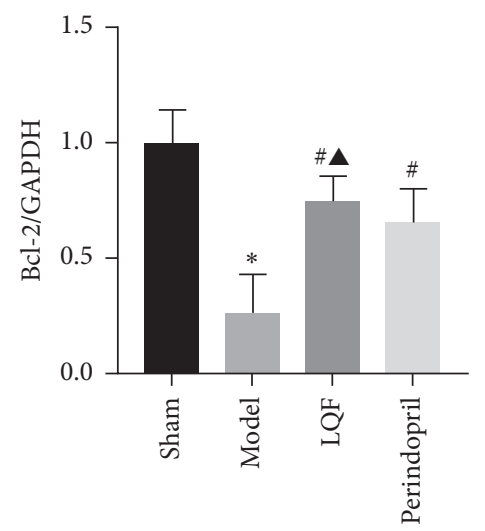

(c)

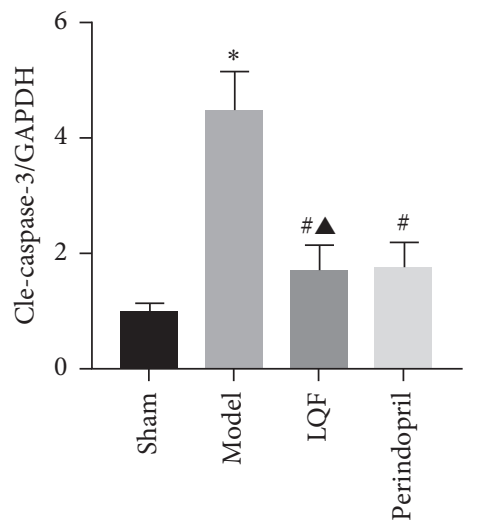

(b)

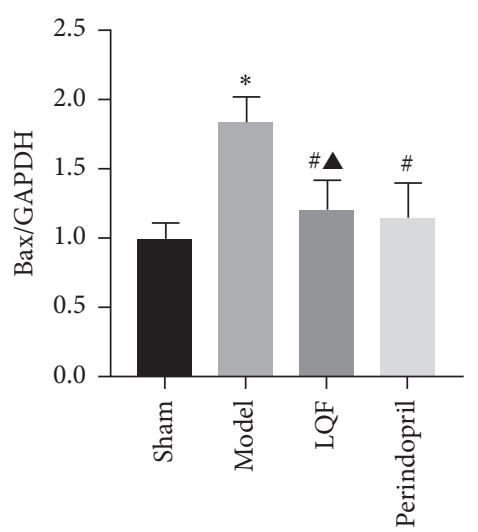

(d)

Figure 5: Continued. 

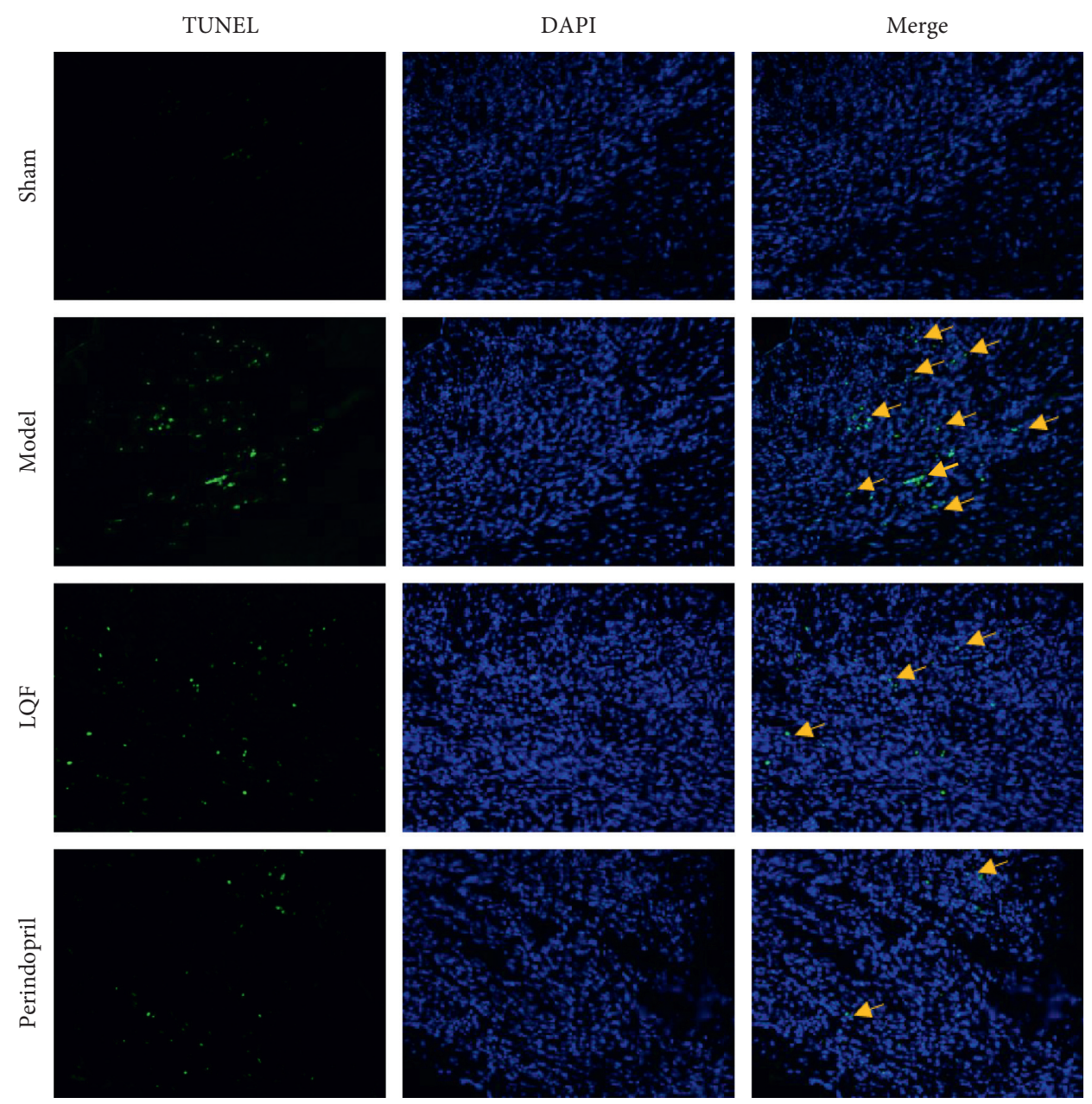

(e)

FIGURE 5: LQF inhibited apoptosis in mice after MI. (a)-(d) Representative western blotting and quantification of apoptosis-related proteins, including Cle-caspase-3, Bcl-2, and Bax, and GAPDH served as a loading control $(n=5) .{ }^{*} P<0.05 v s$. the sham group, ${ }^{\#} P<0.05 v s$. the model group, and $\boldsymbol{\Delta}<0.05$ vs. the perindopril group. (e) TUNEL staining of apoptotic nuclei in cardiac tissues (magnification $200 \times$ ) $(n=3)$.

cardiac dysfunction and the occurrence of an inflammatory response, especially the activation of the NLRP3 inflammasome. The results showed that LQF exerted potent protection against MI and suppressed inflammation in mice. Our results revealed that LQF significantly inhibited the activation of the NLRP3 inflammasome and could be attributable to suppressing the ROS-TXNIP-NLRP3 pathway.

As a macromolecular complex, the NLRP3 inflammasome has been reported to be involved in many CVDs, including atherosclerosis, ischemia/reperfusion (I/R) injury, and HF [30]. A growing body of evidence has revealed that the NLRP3 inflammasome plays a critical role in promoting sterile inflammation through the recognition of danger signals released from dying cells [31]. Therefore, inhibiting the NLRP3 inflammasome is expected to be a novel approach to treating HF [10]. After MI, necrotic cells and stromal debris in the infarcted area produce DAMPs that bind to pattern recognition receptors (PRRs), activate the innate immune system, and produce a strong inflammatory response, leading to an increase in mitochondrial ROS and activation of the NLRP3 inflammasome. Sandanger et al. have found that NLRP3-deficient mice subjected to myocardial I/R injury had a smaller infarct size and preserved cardiac function compared with those of WT mice [32]. Moreover, many active ingredients of LQF, such as carthamin yellow from safflower, astragaloside IV from Astragalus membranaceus, and atractylodesin III from Codonopsis pilosula, have shown anti-inflammatory effects in CVDs [33-35]. In this study, we found that LQF had a significant inhibitory effect on the activation of NLRP3 inflammasomes in MI mice by inhibiting the secretion of IL$1 \beta$ and downregulating the activity of caspase- 1 . The results suggested that LQF can be potentially developed as NLRP3targeting medicine against MI-associated diseases.

The common upstream mechanisms of the NLRP3 inflammasome activation are believed to include intracellular $\mathrm{K}^{+}$outflow, lysosomal breakdown, and production of ROS. Mitochondrial dysfunction leads to changes in the ROS concentration, and the thioredoxin interacting protein separates from the thioredoxin and binds to NLRP3, 
inducing activation of the NLRP3 inflammasome [36]. The findings of numerous previous studies have verified that ROS activates the NLRP3 inflammasome through TXNIP, triggering a rapid cardiac inflammation response [37-39]. In the present study, we observed high expression of mitochondrial ROS and TXNIP in cardiac tissue, which triggered NLRP3 overexpression and cardiac inflammation. However, ROS was suppressed by LQF intervention, which in turn suppressed OS and maintained the TXNIPTrx bond. This bond suppressed TXNIP activity and prevented it from binding with NLRP3, thereby preventing the activation of the inflammasome axis and ameliorating cardiac inflammation and injury. After MI, inflammatory reactions are triggered by DAMPs, which activate macrophages to coordinate inflammation and tissue repair, however, an excessive inflammatory response leads to myocardial apoptosis [40]. Therefore, we examined the apoptosis of cardiomyocytes. Our data showed that Clecaspase- 3 and Bax levels were upregulated in the model group. Conversely, Bcl-2 levels were upregulated significantly after treatment with LQF. The TUNEL assay results also proved the efficacy of LQF against myocardial apoptosis, verifying that one of the mechanisms by which LQF attenuates cardiac apoptosis could be to inhibit the inflammatory response induced by activation of the NLRP3 inflammasome.

In summary, our study demonstrates that LQF prevents ventricular remodeling after MI by regulating NLRP3 inflammasome. The application of Chinese medicine reflects advantages of simplicity and efficiency. Therefore, LQF should be used as a carrier to explore the mechanism of improving ventricular remodeling after MI.

\section{Limitations}

In this study, we only verified the effect of LQF on inhibiting the NLRP3 inflammasome and reducing myocardial apoptosis in animal experiments; therefore, which active component in LQF exerts these specific effects needs to be further clarified in our subsequent studies.

\section{Conclusions}

In summary, we verified that, by inhibiting the production of ROS in cardiac tissue, LQF inhibits NLRP3/ASC/caspase-1/ IL- $\beta$ pathway activation, thereby alleviating cardiac inflammation, fibrosis, and apoptosis. We found that LQF demonstrated excellent performance in protecting cardiac function and ameliorating cardiac inflammation and apoptosis. Interestingly, we also found that perindopril reduced cardiomyocyte apoptosis by inhibiting NLRP3 inflammasome. The present study delineated the therapeutic properties of LQF and its value for subsequent research and clinical applications.

\section{Data Availability}

The data used to support the findings of this study are available from the corresponding author upon request.

\section{Disclosure}

Xiaoqing Zhang and Dandan Zhao are the co-first authors, and Huiyan Qu and Hua Zhou are the co-corresponding authors.

\section{Conflicts of Interest}

The authors declare that there are no conflicts of interest regarding the publication of this article.

\section{Acknowledgments}

This work was supported by the Three-year Action Plan of Shanghai Shenkang Medical Development Center (grant no. SHDC2020CR1053B); the Science and Technology Commission of Shanghai Municipal (grant no. 18401932800); the Shanghai Municipal Health and Family Planning Commission (grant no. ZY-(2018-2020)-CCCX-2003); the Shanghai Medical Leading Talents Program (grant no. 0462015); the Shanghai Shenkang Medical Development Center Emerging Frontier Technology Joint Research Project (grant no. SHDC12018125); the Science and Technology Support Project of Shanghai Science and Technology Commission (grant nos. 20S21901800 and 18401900700); and the Graduate Science and Technology Innovation Program of Shanghai University of Traditional Chinese Medicine (grant no. Y2020014).

\section{References}

[1] F. Triposkiadis, A. Xanthopoulos, and J. Butler, "Cardiovascular aging and heart failure," Journal of the American College of Cardiology, vol. 74, no. 6, pp. 804-813, 2019.

[2] V. Ruddox, I. Sandven, J. Munkhaugen, J. Skattebu, T. Edvardsen, and J. E. Otterstad, "Atrial fibrillation and the risk for myocardial infarction, all-cause mortality and heart failure: a systematic review and meta-analysis," European Journal of Preventive Cardiology, vol. 24, no. 14, pp. 1555-1566, 2017.

[3] N. G. Frangogiannis, "Regulation of the inflammatory response in cardiac repair," Circulation Research, vol. 110, no. 1, pp. 159-173, 2012.

[4] N. G. Frangogiannis, "Cell biological mechanisms in regulation of the post-infarction inflammatory response," Current Opinion in Physiology, vol. 1, pp. 7-13, 2018.

[5] P. R. Hansen, "Role of neutrophils in myocardial ischemia and reperfusion," Circulation, vol. 91, no. 6, pp. 1872-1885, 1995.

[6] Y. Bian, P. Pang, X. Li et al., "CircHelz activates NLRP3 inflammasome to promote myocardial injury by sponging miR-133a-3p in mouse ischemic heart," Journal of Molecular and Cellular Cardiology, vol. 158, pp. 128-139, 2021.

[7] F. Chen, Z. Q. Chen, G. L. Zhong, and J. J. Zhu, "Nicorandil inhibits TLR4/MyD88/NF-kappaB/NLRP3 signaling pathway to reduce pyroptosis in rats with myocardial infarction," Experimental Biology and Medicine (Maywood, NJ), 2021.

[8] S. Toldo and A. Abbate, "The NLRP3 inflammasome in acute myocardial infarction," Nature Reviews: Cardiology, vol. 15, no. 4, pp. 203-214, 2018.

[9] M. Takahashi, "Role of NLRP3 inflammasome in cardiac inflammation and remodeling after myocardial infarction," Biological and Pharmaceutical Bulletin, vol. 42, no. 4, pp. 518-523, 2019. 
[10] Y. Tong, Z. Wang, L. Cai, L. Lin, J. Liu, and J. Cheng, "NLRP3 inflammasome and its central role in the cardiovascular diseases," Oxidative Medicine and Cellular Longevity, vol. 2020, Article ID 4293206, 8 pages, 2020.

[11] R. Gao, H. Shi, S. Chang et al., "The selective NLRP3inflammasome inhibitor MCC950 reduces myocardial fibrosis and improves cardiac remodeling in a mouse model of myocardial infarction," International Immunopharmacology, vol. 74, Article ID 105575, 2019.

[12] Y. Wang, B. Gao, and S. Xiong, "Involvement of NLRP3 inflammasome in CVB3-induced viral myocarditis," American Journal of Physiology: Heart and Circulatory Physiology, vol. 307, no. 10, pp. H1438-H1447, 2014.

[13] N. Lapointe, C. Blais, A. Adam et al., "Comparison of the effects of an angiotensin-converting enzyme inhibitor and a vasopeptidase inhibitor after myocardial infarction in the rat," Journal of the American College of Cardiology, vol. 39, no. 10, pp. 1692-1698, 2002.

[14] M. A. Pfeffer, E. Braunwald, L. A. Moyé et al., "Effect of captopril on mortality and morbidity in patients with left ventricular dysfunction after myocardial infarction," New England Journal of Medicine, vol. 327, no. 10, pp. 669-677, 1992.

[15] T. Yang, H. Qu, X. Song et al., "Luhong granules prevent ventricular remodelling after myocardial infarction by reducing the metabolites TMAO and LPS of the intestinal flora," Evidence-Based Complementary and Alternative Medicine, vol. 201910 pages, 2019.

[16] L. Li, D. Yang, J. Li et al., "Investigation of cardiovascular protective effect of Shenmai injection by network pharmacology and pharmacological evaluation," BMC Complementary Medicine and Therapies, vol. 20, no. 1, p. 112, 2020.

[17] H. Mao, P. Su, W. Qiu, L. Huang, H. Yu, and Y. Wang, "The use of masson's trichrome staining, second harmonic imaging and two-photon excited fluorescence of collagen in distinguishing intestinal tuberculosis from crohn's disease," Colorectal Disease, vol. 18, no. 12, pp. 1172-1178, 2016.

[18] K. J. Livak and T. D. Schmittgen, "Analysis of relative gene expression data using real-time quantitative PCR and the 2- $\Delta \Delta$ CT method," Methods, vol. 25, no. 4, pp. 402-408, 2001.

[19] C. Chen, C. Qin, H. Qiu et al., "Deep learning for cardiac image segmentation: a review," Frontiers in Cardiovascular Medicine, vol. 7, no. 25, 2020.

[20] S. S. Romańczyk M and K. Łabuzek, "Natriuretic peptides in diagnostics and therapy," Polski Merkuriusz Lekarski, vol. 48, no. 287, pp. 370-374, 2020.

[21] M. A. Pfeffer, "Enhancing cardiac protection after myocardial infarction: rationale for newer clinical trials of angiotensin receptor blockers," American Heart Journal, vol. 139, no. 2, pp. 23-28, 2000.

[22] P. Raj, B. M. Aloud, X. L. Louis, L. Yu, S. Zieroth, and T. Netticadan, "Resveratrol is equipotent to perindopril in attenuating post-infarct cardiac remodeling and contractile dysfunction in rats," The Journal of Nutritional Biochemistry, vol. 28, pp. 155-163, 2016.

[23] S. Li, S. Li, X. Hao, Y. Zhang, and W. Deng, "Perindopril and a galectin-3 inhibitor improve ischemic heart failure in rabbits by reducing gal-3 expression and myocardial fibrosis," Frontiers in Physiology, vol. 10, 2019.

[24] J. Bauersachs, "Regulation of myocardial fibrosis by MicroRNAs," Journal of Cardiovascular Pharmacology, vol. 56, no. 5, pp. 454-459, 2010.
[25] X. Huang, Y. Liu, X. Yang et al., "NH4Cl treatment prevents doxorubicin-induced myocardial dysfunction in vivo," Life Sciences, vol. 227, pp. 94-100, 2019.

[26] M. J. M. Silvis, E. J. Demkes, A. T. L. Fiolet et al., "Immunomodulation of the NLRP3 inflammasome in atherosclerosis, coronary artery disease, and acute myocardial infarction," Journal of Cardiovascular Translational Research, vol. 14, no. 1, pp. 23-34, 2021.

[27] A. P. Kalogeropoulos, V. V. Georgiopoulou, and J. Butler, "From risk factors to structural heart disease: the role of inflammation," Heart Failure Clinics, vol. 8, no. 1, pp. 113123, 2012.

[28] P. M. Ridker, B. M. Everett, T. Thuren et al., "Antiinflammatory therapy with canakinumab for atherosclerotic disease," New England Journal of Medicine, vol. 377, no. 12, pp. 1119-1131, 2017.

[29] J. C. Tardif, S. Kouz, D. D. Waters et al., "Efficacy and safety of low-dose colchicine after myocardial infarction," New England Journal of Medicine, vol. 381, no. 26, pp. 2497-2505, 2019.

[30] N. An, Y. Gao, Z. Si et al., "Regulatory mechanisms of the NLRP3 inflammasome, a novel immune-inflammatory marker in cardiovascular diseases," Frontiers in Immunology, vol. 10, 2019.

[31] B. Butts, R. A. Gary, S. B. Dunbar, and J. Butler, "The importance of NLRP3 inflammasome in heart failure," Journal of Cardiac Failure, vol. 21, no. 7, pp. 586-593, 2015.

[32] O. Sandanger, T. Ranheim, L. E. Vinge et al., "The NLRP3 inflammasome is up-regulated in cardiac fibroblasts and mediates myocardial ischaemia-reperfusion injury," Cardiovascular Research, vol. 99, no. 1, pp. 164-174, 2013.

[33] H. W. Tong Zhang, M. Lu, Z. Kun, J. Yin, and L. Yang, "Astragaloside IV prevents myocardial hypertrophy induced by mechanical stress by activating autophagy and reducing inflammation," American Journal of Translational Research, vol. 12, no. 9, pp. 5332-5342, 2020.

[34] C. Y. Mingying Cao, Z. Yao, X. Gao, and S. Wu, “Atractylodesin III maintains mitochondrial function and inhibits caspase-3 activity to reverse apoptosis of cardiomyocytes in AMI rats," International Journal of Clinical and Experimental Pathology, vol. 12, no. 1, pp. 198-204, 2019.

[35] Q. Y. Lu, J. Q. Ma, Y. Y. Duan et al., "Carthamin yellow protects the heart against ischemia/reperfusion injury with reduced reactive oxygen species release and inflammatory response," Journal of Cardiovascular Pharmacology, vol. 74, no. 3, pp. 228-234, 2019.

[36] R. Zhou, A. Tardivel, B. Thorens, I. Choi, and J. Tschopp, "Thioredoxin-interacting protein links oxidative stress to inflammasome activation," Nature Immunology, vol. 11, no. 2, pp. 136-140, 2010.

[37] D. S. Wang, L. Y. Yan, D. Z. Yang et al., "Formononetin ameliorates myocardial ischemia/reperfusion injury in rats by suppressing the ROS-TXNIP-NLRP3 pathway," Biochemical and Biophysical Research Communications, vol. 525, no. 3, pp. 759-766, 2020.

[38] Y. Liu, K. Lian, L. Zhang et al., "TXNIP mediates NLRP3 inflammasome activation in cardiac microvascular endothelial cells as a novel mechanism in myocardial ischemia/ reperfusion injury," Basic Research in Cardiology, vol. 109, no. 5, p. 415, 2014.

[39] Y. Wu, F. He, Y. Li et al., "Effects of shizhifang on NLRP3 inflammasome activation and renal tubular injury in hyperuricemic rats," Evidence-Based Complementary and 
Alternative Medicine, vol. 2017, Article ID 7674240, 19 pages, 2017.

[40] J. H. Erlich, E. M. Boyle, J. Labriola et al., "Inhibition of the tissue factor-thrombin pathway limits infarct size after myocardial ischemia-reperfusion injury by reducing inflammation," The American Journal of Pathology, vol. 157, no. 6, pp. 1849-1862, 2000. 